\title{
Urban Versus Rural: A Cross-Sectional Study of a Successful Peruvian Global Health Medical Mission
}

\author{
Franklin N, BA ${ }^{1}$ \& Quian A, BS, MA ${ }^{1}$, Choi H, $\mathrm{MD}^{2}$, Valdivieso M, $\mathrm{MD}^{3}$, \\ Lam SK, MD, MPH, MS $^{2 *}$ \\ ${ }^{1}$ Case Western Reserve University School of Medicine, 9501 Euclid Ave, Cleveland, OH 44106, US \\ ${ }^{2}$ Cleveland Clinic, 9500 Euclid Ave, S1-20, Cleveland, OH 44195, US \\ ${ }^{3}$ Peruvian American Medical Society, Chincha Alta
}

*Corresponding Author: Lam SK, Cleveland Clinic Lerner College of Medicine, Case Western Reserve University School of Medicine, OH, USA. Email: LAMS2@ccf.org

\begin{abstract}
Background: The purpose of this study is to characterize the health conditions of two Peruvian populations in Chincha Alta and Sacred Valley, during a short-term medical mission in order to improve future global health efforts. Existing literature on the disease prevalence in these specific communities have not been previously reported.

Methods: The study team consisted of medical students and faculty from a United States-based academic medical institution. The study site in CA was a modern local outpatient clinic and in SV, health posts in various small towns. This is a cross-sectional study that utilized convenience sampling of patients who sought care at previously established and temporary health clinics. Descriptive and comparative statistics were used.

Results: Over three weeks, 420 patients were seen (CA $n=197 ;$ SV n=223). Among adult patients, musculoskeletal $(P<.001)$, head/neck $(P<.001)$, neurologic $(P<.001)$, gastrointestinal $(P<.001)$, and genitourinary $(P=.004)$ complaints were more common in $S V$ than $C A$. Adult diagnoses of chronic musculoskeletal pain $(P<.001)$, genitourinary infection $(P=.03)$, gastritis $(P=.01)$, and dehydration $(P=.02)$ were more frequent in $S V$ with lower respiratory infection $(P=.002)$ and obesity $(P=.009)$ being more frequent in $C A$.
\end{abstract}

Conclusions: This study demonstrates the need for future STMMs to these Peruvian communities to refine prevention and management strategies.

Keywords: Epidemiology, global health, rural, urban, Peru, medical mission

\section{INTRODUCTION}

In Peru, the data on health conditions of limited resource and lower-income areas are inconsistent and research is needed to illuminate health access, health conditions, and health needs of the local communities. Existing literature on demographics and disease prevalence in the Chincha Alta (CA) and Sacred Valley (SV) communities in Peru are sparse; most Englishlanguage literature about Peru focus on global health outreach projects or specific infectious diseases. [1-3] Without extensive information, it is difficult for short-term medical mission (STMM) teams to provide specific and targeted clinical and public health interventions.

The Peruvian government (MINSA) provides limited health care to approximately $60 \%$ of the population, $30 \%$ are covered by Peruvian employee insurance (ESSALUD), and police and military hospital coverage and private insurance account for the remaining 10\%. [4] The average life expectancy is $73 / 78$ (male/female) years and the most common causes of death in adults are all cancers, influenza and pneumonia, and coronary heart disease. [5, 6] The infant mortality rate in 2015 was 15 deaths per 1,000 live births with the highest rates of mortality occurring in mountain and jungle areas. [7] In children aged five to nine years, the leading causes of mortality in 2014 were malignant neoplasms of lymphoid and hematopoietic tissue, influenza and pneumonia, septicemia, congenital malformations and chromosome abnormalities, and accidental threats to breathing. For adolescents aged 12 to 17 years, the primary complaints for outpatient 
visits included acute respiratory infections, dental caries, and malnutrition or nutritional deficiencies in 2011. [7, 8] Although national data is helpful for public health purposes, it may miss local trends and a specific community's demographics and health needs are necessary for treating patients at the local level.

CA is an urban coastal area and the capital of the Chincha region. Similar to many urban environments, CA's population may see higher rates of respiratory conditions related to pollution and poor air quality, such as asthma. [9, 10] Private clinics and local hospitals are available in CA for access to primary care.

In the SV area of Peru, the majority Quechuaspeaking population is employed in traditional farming or the tourism industry. SV is located in the remote Andean highlands, consisting of mountainous villages, with limited access to developed roads, water, and electricity. Primary care is provided in the form of local puestos de salud, or health posts. Unique health considerations to this population include the effects of altitude and manual labor, potentially leading to higher rates of musculoskeletal pain. [11] Anemia in Peruvian children is also more common at high altitudes compared to lower altitudes. [12]

The World Health Organization (WHO) notes that rural areas continue to face inequality in lower distribution of health workers compared to coastal areas. [4] The goals of this preliminary study are to characterize the health conditions of the local populations of CA and SV. The hypothesis of this study is that there will be differences in disease conditions between the two communities.

\section{MATERIALS AND MeTHOdS}

\subsection{Study Design}

This is a cross-sectional study comparing the patients evaluated in CA and SV during an annual STMM. Institutional Review Board approval was received by the home institution and both CA and SV sites.

The data in CA were collected over 10 days during the winter season. The home institution collaborated with a modern local outpatient clinic that operates year-round. The visiting team consisted of a family physician, pulmonologist, pediatrician, cardiologist, and medical students. The clinic offered a variety of services, including routine radiology, laboratory services and ultrasound.

The data in SV were collected over five days during the winter season. The health care team in
SV was composed of internists, pediatricians, an orthopedist, and medical students. The PHOP team collaborated with a non-profit organization, which organized the daily locations for the team. Each day, the health care team visited a different rural health post located among the numerous pueblos in Sacred Valley, where members of the nearby pueblos would visit the clinic. Interpreters were used to interpret Quechua, the native indigenous language, to Spanish. Simple tests, such as vital signs, fingerstick glucose test, hemoglobin test, and urine dipstick were available.

Convenience sampling of all patients seen by a physician at the clinics were included in the study. The health care team collected the patient's data using a case report form in real time during the clinic visit to minimize recall bias. Patient health information collected included demographics, chief complaint, diagnosis, and treatment.

\subsection{Statistical Analysis}

Data between CA and SV were stratified by age to either pediatric $(<18)$ or adult groups and then compared. The seven patients who did not report their age were excluded. Data from the case report forms was managed with REDCap electronic database (NIH). (23) Statistical analyses were completed using JMP software (SAS Institute, Cary NC). Categorical variables were compared using the Chi-square test. Continuous variables were tested for normality using the Shapiro-Wilk test, and if there was not a normal distribution, the Wilcoxon (Mann-Whitney) test was used for comparison.

\section{RESULTS AND DISCUSSION}

A total number of 420 patients were seen, 197 at $\mathrm{CA}$ and 233 at SV (Table 1). The median age of patients from CA was 25 years (IQR 5, 60) and from SV was 27.5 years (IQR 5, 53), with no difference in age distribution $(P=.60)$. The pediatric age group consisted of 165 total patients, 80 were from CA (41\%) and 85 were from SV (39\%). A total of 248 adult patients were seen, 115 from CA (59\%) and 133 from SV (61\%). The majority of patients were female at both sites (CA 58\%, SV 61\%). Spanish language was predominately spoken in CA, whereas in SV, both Spanish and Quechua were spoken. Herbal medication use was more common in SV than in CA $(P<.001)$. From the patient-reported past medical history, hypertension was more frequent in CA $(P=.02)$, while gastritis and anemia were more frequent in $\mathrm{SV}(P=.04$ and $P=.02$, respectively). 
Table1. Patient Demographics

\begin{tabular}{|c|c|c|c|c|c|}
\hline & & $\begin{array}{l}\text { Both Sites } \\
N=420 n(\%)\end{array}$ & $\begin{array}{l}\text { Chincha Alta } \\
N=197 n(\%)\end{array}$ & $\begin{array}{l}\text { Sacred Valley } \\
N=233 n(\%)\end{array}$ & $P$ value \\
\hline \multirow[t]{3}{*}{ Gender } & Female & $244(58)$ & $109(55)$ & $135(61)$ & .54 \\
\hline & Male & $145(35)$ & $72(37)$ & $73(33)$ & \\
\hline & Not reported & $31(7)$ & $16(8)$ & $15(7)$ & \\
\hline \multirow[t]{11}{*}{ Age } & $\leq 4$ years & $92(22)$ & $42(21)$ & $50(21)$ & \\
\hline & $5-14$ & $68(16)$ & $38(19)$ & $30(13)$ & \\
\hline & $15-24$ & $30(7)$ & $13(7)$ & $17(7)$ & \\
\hline & $25-34$ & $48(11)$ & $15(8)$ & $33(14)$ & \\
\hline & $35-44$ & $30(7)$ & $10(5)$ & $20(9)$ & \\
\hline & $45-54$ & $34(8)$ & $18(9)$ & $16(7)$ & \\
\hline & $55-64$ & $35(8)$ & $18(9)$ & $17(7)$ & \\
\hline & $65-74$ & $39(9)$ & $25(13)$ & $14(6)$ & \\
\hline & $75-84$ & $23(5)$ & $11(6)$ & $12(5)$ & \\
\hline & $85-94$ & $14(3)$ & $5(3)$ & $9(4)$ & \\
\hline & $>95$ & $0(0)$ & $0(0)$ & $0(0)$ & \\
\hline \multirow[t]{3}{*}{ Language } & Spanish & $325(77)$ & $180(91)$ & $145(62)$ & $<.001 *$ \\
\hline & Quechua & $89(21)$ & $1(1)$ & $88(38)$ & $<.001 *$ \\
\hline & English & $1(0)$ & $1(1)$ & $0(0)$ & .47 \\
\hline Medication & Acute & $61(15)$ & $28(14)$ & $33(14)$ & .86 \\
\hline \multirow[t]{3}{*}{ Use } & Chronic & $39(9)$ & $17(9)$ & $22(9)$ & .66 \\
\hline & Herbal & $51(12)$ & $1(1)$ & $50(21)$ & $<.001 *$ \\
\hline & None & $136(32)$ & $65(33)$ & $71(30)$ & .80 \\
\hline Patient - & None & $165(40)$ & $80(41)$ & $85(38)$ & .60 \\
\hline Reported & Not reported & $109(26)$ & $39(20)$ & $70(31)$ & $.04 *$ \\
\hline Past & Hypertension & $37(9)$ & $24(12)$ & $13(6)$ & $.02 *$ \\
\hline Medical & Gastritis & $31(7)$ & $9(5)$ & $22(10)$ & $.04 *$ \\
\hline History & Anemia & $20(5)$ & $4(2)$ & $16(7)$ & $.02 *$ \\
\hline \multicolumn{2}{|c|}{$* P$ value $<.05$} & & & & \\
\hline
\end{tabular}

Among the pediatric population, head, ears, eyes, nose \& throat (HEENT); musculoskeletal (MSK); and genitourinary (GU) chief complaints presented more frequently in $\mathrm{SV}$ than $\mathrm{CA}$ ( $P=.009, P=.003, P=.03$, respectively). All other pediatric chief complaints were similar between the two sites, with the most common chief complaints being respiratory, systemic, and gastrointestinal (GI). Within the adult population, MSK $(P<.001)$, HEENT $(P<.001)$, neurologic $(P<.001)$, GI $(P<.001)$, and GU $(P=.004)$ complaints were substantially more frequent in SV than CA. The most common adult complaints in CA were respiratory, MSK, and systemic; whereas in SV were MSK, HEENT, and respiratory.

There were no differences observed within the pediatric population for types of diagnosis between sites. In $\mathrm{CA}$, the most frequently Table2. Adult Patient Diagnoses

\begin{tabular}{|c|c|c|c|c|}
\hline & $\begin{array}{l}\text { Both Sites } \\
N=248 \\
n(\%)\end{array}$ & $\begin{array}{l}\text { Chincha Alta } \\
N=115 \\
n(\%)\end{array}$ & $\begin{array}{l}\text { Sacred Valley } \\
N=133 \\
n(\%)\end{array}$ & $P$ value \\
\hline Chronic MSK pain & $58(23)$ & $7(6)$ & $51(38)$ & $<.001 *$ \\
\hline Upper respiratory infection & $32(13)$ & $16(14)$ & $16(12)$ & .66 \\
\hline Hypertension & $23(9)$ & $15(13)$ & $8(6)$ & .06 \\
\hline Genitourinary infection & $19(8)$ & $4(3)$ & $15(11)$ & $.03^{*}$ \\
\hline
\end{tabular}

observed pediatric diagnoses were upper respiratory infection (URI), intestinal parasites, dermatologic disease, lower respiratory infection (LRI), and gastritis. In SV, URI, dermatologic disease, well-child visit, parasite infection, dehydration and developmental delay were the most frequently observed pediatric diagnoses.

Considerable adult diagnosis differences were observed between the two sites, with chronic MSK pain $(P<.001)$, GU infection $(P=.03)$, gastritis $(P=.01)$ and dehydration $(P=.02)$ being more common in SV; whereas, LRI $(P=.002)$, obesity $(P=.009)$ and inconclusive diagnosis $(P=.03)$ were more common in CA (Table 2). In CA, the most frequently observed adult diagnoses were URI, hypertension, and LRI. In $\mathrm{SV}$, chronic musculoskeletal pain, URI, GU infection, and gastritis were the most common adult diagnoses. 


\begin{tabular}{|l|l|l|l|l|}
\hline Gastritis & $18(7)$ & $3(3)$ & $15(11)$ & $.01^{*}$ \\
\hline Acute MSK pain & $17(7)$ & $7(6)$ & $10(8)$ & .66 \\
\hline Headache disorder & $16(6)$ & $6(5)$ & $10(8)$ & .46 \\
\hline Lower respiratory infection & $15(6)$ & $13(11)$ & $2(2)$ & $.002^{*}$ \\
\hline Dehydration & $10(4)$ & $1(1)$ & $9(7)$ & $.02^{*}$ \\
\hline Diabetes & $9(4)$ & $7(6)$ & $2(2)$ & .09 \\
\hline Asthma & $9(4)$ & $5(4)$ & $4(3)$ & .74 \\
\hline Dermatologic issue & $8(3)$ & $5(4)$ & $3(2)$ & .48 \\
\hline Inconclusive & $8(3)$ & $7(6)$ & $1(1)$ & $.03^{*}$ \\
\hline Mental health disorder & $7(3)$ & $3(3)$ & $4(3)$ & 1.00 \\
\hline Obesity & $6(2)$ & $6(5)$ & $0(0)$ & $.009^{*}$ \\
\hline$* P$ value <.05 & & & & \\
\hline
\end{tabular}

The treatments for pediatric patients differed markedly between the two sites, with medication prescription $(P=.001)$ and further testing $(P=.03)$ utilized more often at CA (Table 3$)$. Both sites mainly used medication prescriptions and patient education as their main treatment modalities.

Table3. Pediatric Patient Treatments

\begin{tabular}{|l|l|l|l|l|}
\hline & $\begin{array}{l}\text { Both Sites } \\
\mathbf{N = 1 6 5} \boldsymbol{n}(\boldsymbol{\%})\end{array}$ & $\begin{array}{l}\text { Chincha Alta } \\
\boldsymbol{N = 8 0} \boldsymbol{n}(\boldsymbol{\%})\end{array}$ & $\begin{array}{l}\text { Sacred Valley } \\
\boldsymbol{N = 8 5} \boldsymbol{n}(\boldsymbol{\%})\end{array}$ & $\boldsymbol{P}$ value \\
\hline Medication prescription & $114(69)$ & $65(81)$ & $49(58)$ & $<.001^{*}$ \\
\hline Patient education & $88(53)$ & $41(51)$ & $47(55)$ & .60 \\
\hline Referral & $16(10)$ & $4(5)$ & $12(14)$ & .06 \\
\hline Further testing & $8(5)$ & $7(9)$ & $1(1)$ & $.03^{*}$ \\
\hline Follow-up required & $3(2)$ & $1(1)$ & $2(2)$ & 1.00 \\
\hline No treatment required & $3(2)$ & $0(0)$ & $3(4)$ & .25 \\
\hline Procedure & $1(1)$ & $1(1)$ & $0(0)$ & .48 \\
\hline$* \boldsymbol{P}$ value <.05 & & & \\
\hline
\end{tabular}

For adult patients, the treatment at both sites utilized medication prescription; however, SV focused more on patient education $(P<.001)$ and referral $(P=.01)$, compared to $\mathrm{CA}$, which had a

higher occurrence of further testing $(P<.001)$ and no treatment $(P=.02)$ (Table 4). Analgesics $(P=.004)$ and antacids $(P=.008)$ were prescribed more often in $\mathrm{SV}$ and antibiotics $(P=.003)$ were more frequently prescribed in $\mathrm{CA}$. amount of antibiotics were prescribed in CA compared to $\mathrm{SV} \quad(P<.001)$. The majority of medications prescribed in $\mathrm{CA}$ and $\mathrm{SV}$ were analgesics, antibiotics, antiparasite medications, NSAIDs, and antihistamines.

Table4. Adult Patient Treatments

\begin{tabular}{|l|l|l|l|l|}
\hline & $\begin{array}{l}\text { Both Sites } \\
\mathbf{N = 2 4 8} \boldsymbol{n}(\boldsymbol{\%})\end{array}$ & $\begin{array}{l}\text { Chincha Alta } \\
\boldsymbol{N = 1 1 5} \boldsymbol{n}(\boldsymbol{\%})\end{array}$ & $\begin{array}{l}\text { Sacred Valley } \\
\boldsymbol{N = 1 3 3} \boldsymbol{n}(\boldsymbol{\%})\end{array}$ & $\boldsymbol{P}$ value \\
\hline Medication prescription & $166(67)$ & $81(70)$ & $85(64)$ & .28 \\
\hline Patient education & $120(48)$ & $42(37)$ & $78(59)$ & $<.001^{*}$ \\
\hline Referral & $37(15)$ & $10(9)$ & $27(20)$ & $.01^{*}$ \\
\hline Further testing & $32(13)$ & $25(22)$ & $7(5)$ & $<.001^{*}$ \\
\hline Follow-up required & $17(7)$ & $11(10)$ & $6(5)$ & .12 \\
\hline No treatment required & $5(2)$ & $5(4)$ & $0(0)$ & $.02^{*}$ \\
\hline Procedure & $5(2)$ & $3(3)$ & $2(2)$ & .67 \\
\hline$* \boldsymbol{P}$ value $<.05$ & & & & \\
\hline
\end{tabular}

\section{Conclusions}

The purpose of this study was to characterize the health of the local Peruvian populations, specific to urban $\mathrm{CA}$ and rural $\mathrm{SV}$, to help direct future health care efforts. Overall, both pediatric and adult populations had high rates of URIs. There were notable differences in common adult disease conditions between the two sites, with MSK pain, GU infection, and dehydration being more predominant in $\mathrm{SV}$, in contrast to LRI and obesity being more common in CA. Treatment
Among the medications prescribed, a greater

differences included more medication dispensing and testing in $\mathrm{CA}$ compared to $\mathrm{SV}$, perhaps due to the increased availability of resources at the CA clinic.

This study is limited by a number of factors around the short period of study and convenience sampling. For both locations, all patients were walk-in. Patients were notified of the team specialties through written signage outside both clinic locations and by word of mouth. The types of patients who sought care and the diagnoses may have been affected by the specialties of the 
physicians. The availability of pediatric specialists may have also attracted a greater number of pediatric patients, specifically school classes, many of whom only sought well-child visits with no chief complaints.

The basic structures of the clinics in $\mathrm{CA}$ and $\mathrm{SV}$ were markedly different. In CA, the PHOP team worked at an established clinic that had its own permanent facilities and access to resources, i.e. $\mathrm{x}$-rays, labs, pharmacy, etc. In contrast, SV clinics were conducted at five different locations utilizing local health posts or empty school or community rooms and all supplies were brought by the team. Although available physicians provided care to the best of their clinical abilities, the lack of laboratory testing limited the accuracy of some diagnoses.

Based on this data, it is apparent that future medical teams need to take the local culture into account. Besides a language barrier, there may be medical concepts that are difficult to interpret in the Quechua language and culture. Additionally, the properties and effects of herbal medicines are still not well understood and could cause drugdrug interactions.

Amongst patients in SV as compared to CA, there were more reported previous cases of anemia and gastritis, suggesting that these conditions are more commonly diagnosed by local practitioners. Findings of higher rates of anemia in higher altitude areas correlate to the results found by Amerson et al., who found that $47.5 \%$ of 160 participants aged seven months to six years from a high altitude region of Peru had anemia. [12] Both poor nutrition and sanitation likely factors into the high rates of anemia, suggested by common complaints of gastritis and parasitic disorders. Several studies estimate that $80 \%$ of the population is infected with $H$. pylori in Latin America. [13, 14]

In both locations, upper respiratory infections were very common among both pediatric and adult populations, which is expected due to the winter season. These findings may be explained by seasonal increases in flu rates. [15]

One difference in both complaints and diagnoses between the two sites was in musculoskeletal conditions. Vega-Hinojoso et al. also found an increased prevalence of MSK complaints in high altitude Peru, reporting that $12 \%$ of 1,095 people sampled had MSK pain. [11] These findings likely relate to many of the patients' occupations in manual labor and agricultural fields, which are well-established causes of MSK complaints. [1619]
Occupational customs in SV may impact hydration status. When asked, some patients reported that while doing agricultural work, it was very inconvenient to carry water and stay adequately hydrated. A study in Florida noted that $53 \%$ of 192 workers were dehydrated preshift, increasing to $81 \%$ post-shift, corroborating the difficulties of hydration during fieldwork. [20] Similarly in Nicaragua, a cross-sectional study showed low fluid intake, dehydration and dysuria to be common among the small-scale farmers, which ultimately may impact chronic kidney function. [21]

Associations between urban areas and noncommunicable diseases, such as hypertension and diabetes, as well as social behaviors, such as smoking and drinking, have been discussed by various studies of the PERU MIGRANT cohort. [22-24] This cohort surveyed 154 rural residents, 520 rural-to-urban migrants, and 173 urban residents to assess for cardiovascular disease risk factors and major noncommunicable diseases. (24) The PERU MIGRANT cohort showed 29\% of the urban residents had hypertension, compared to $11 \%$ of the rural residents. Higher BMIs in the urban population were noted as well. [23] Our study similarly found a higher occurrence of hypertension history and obesity diagnoses in the urban CA cohort, as compared to the rural SV cohort.

Future research should look further into the differences between urban and rural health landscapes in South American countries. While this research suggests similar results to past studies showing higher rates of noncommunicable diseases in urban areas, further investigation into lifestyle differences may reveal areas of interest for public health efforts. This is a preliminary study that should continue longitudinally as this STMM returns to Peru annually. While vitals such as height, weight, blood pressure, and heart rate were often obtained during care, they were not consistently recorded and thus could not be included in this study.

With regards to the pediatric population, future studies may benefit from collecting more information on vaccinations, growth charts, and nutrition. While this data was outside the scope of our study, longitudinal collection of such information could greatly benefit pediatric care in rural areas.

In hopes of maximizing care and safety of patients while minimizing cross-cultural misunderstandings, several groups have 
published recommendations for STMMs. [2, 2632] Lasker et al. enumerates themes across several guidelines, including preparation of volunteers, partnership with host organization as well as needs assessment, sustainability, legal and ethical conduct, and continued evaluation. [32] Without including these considerations, STMMs run the risk of wasting resources, or even negatively impacting the healthcare of the local population.

This study encourages other STMM groups to consider the local needs of their specific population and demonstrates that collection of this type of data is both feasible and beneficial to long-term healthcare efforts. For these locations, future STMM groups should target prevention and management efforts towards infectious disease at both sites, hydration techniques, and physical therapy in SV and non-communicable diseases, such as hypertension and metabolic syndrome, in CA.

\section{ACKNOWLEDGMENTS}

We would like to thank all the physicians, nurses, residents, and medical students who participated in this trip. We would like to thank the Hearst Summer Research Fellowship for partially supporting this project. We would like to thank the two Quechua-Spanish translators for their invaluable service. Lastly, we thank all the patients and local hosts for their trust and hospitality.

\section{REFERENCES}

[1] Moro P. L. et al., Short report: endemic focus of cystic echinococcosis in a coastal city of Peru. Am. J. Trop. Med. Hyg. 71, 327-329 (2004).

[2] Geen O., Pumputis A., Kochi C., Costa A., and Stobbe K., Assessing the Short-Term Global Health Experience: A Cross-Sectional Study of Demographics, Socioeconomic Factors, and Disease Prevalence. The American Journal of Tropical Medicine and Hygiene 97, 596-601 (2017).

[3] Acosta, G. J. et al., Diarrheagenic Escherichia coli: Prevalence and Pathotype Distribution in Children from Peruvian Rural Communities. Am. J. Trop. Med. Hyg. 95, 574-579 (2016).

[4] Global health workforce alliance. WHO|Peru. WHO http://www.who.int/workforcealliance/ countries/per /en/.

[5] WHO|Peru.WHO http://www.who.int/ countries /per/en/.

[6] WHO. WHO / WHO Mortality Database. WHO https://www.who.int/healthinfo/mortality_data/ en/.

[7] Pan American Health Organization. Health in the Americas. (2017).
[8] Pan American Health Organization. Country Report: Peru. Health in the Americas 2017 https://www.paho.org/salud-en-las-americas$2017 / ? \mathrm{p}=3232$ (2017).

[9] Underhill, L. J. et al., Association of Roadway Proximity with Indoor Air Pollution in a PeriUrban Community in Lima, Peru. Int J Environ Res Public Health 12, 13466-13481 (2015).

[10] Robinson, C. L. et al., The Peru Urban versus Rural Asthma (PURA) Study: methods and baseline quality control data from a crosssectional investigation into the prevalence, severity, genetics, immunology and environmental factors affecting asthma in adolescence in Peru. BMJ Open 2, (2012).

[11] Vega-Hinojosa O., Cardiel M. H., and OchoaMiranda P., Prevalence of musculoskeletal manifestations and related disabilities in a Peruvian urban population living at high altitude. COPCORD Study. Stage I. Reumatol Clin (2017).

[12] Amerson, R., Duggan L., Glatt M., Ramsey K., and Baker J., Assessment of Anemia Levels in Infants and Children in High Altitude Peru. Global Journal of Health Science 9, 87 (2017).

[13] Valdivieso M. et al., Clinical, Epidemiologic, and Genomic Studies (SWOG S1119) of Helicobacter Pylori in Lima, Peru: Role of Contaminated Water. Journal of Cancerology 3, 12 (2016).

[14] Greenberg E. R. et al., 14-day triple, 5-day concomitant, and 10-day sequential therapies for Helicobacter pylori infection in seven Latin American sites: a randomised trial. Lancet 378, 507-514 (2011).

[15] WHO|FluNet. WHO http://www.who.int/ influenza/gisrs_laboratory/flunet/en/.

[16] Xiao H., McCurdy S. A., Stoecklin-Marois M. T., Li C. S., and Schenker M. B., Agricultural work and chronic musculoskeletal pain among Latino farm workers: the MICASA study. Am. J. Ind. Med. 56, 216-225 (2013).

[17] Lee, S. J., Tak S., Alterman T., and Calvert G. M., Prevalence of musculoskeletal symptoms among agricultural workers in the United States: an analysis of the National Health Interview Survey, 2004-2008. J Agromedicine 19, 268280 (2014).

[18] Meucci R. D., Fassa A. G., Faria N. M. X., and Fiori N. S., Chronic low back pain among tobacco farmers in southern Brazil. Int J Occup Environ Health 21, 66-73 (2015).

[19] Osborne A. et al., Risk factors for musculoskeletal disorders among farm owners and farm workers: a systematic review. Am. J. Ind. Med. 55, 376-389 (2012). 
[20] Mix J. et al., Hydration Status, Kidney Function, and Kidney Injury in Florida Agricultural Workers. J. Occup. Environ. Med. 60, e253e260 (2018).

[21] Wesseling C. et al., Heat stress, hydration and uric acid: a cross-sectional study in workers of three occupations in a hotspot of Mesoamerican nephropathy in Nicaragua. BMJ Open 6, e011034 (2016).

[22] Ruiz-Alejos A. et al., Addressing the impact of urban exposure on the incidence of type 2 diabetes mellitus: The PERU MIGRANT Study. Sci Rep 8, 5512 (2018).

[23] Miranda J. J., Gilman R. H., and Smeeth L., Differences in cardiovascular risk factors in rural, urban and rural-to-urban migrants in Peru. Heart 97, 787-796 (2011).

[24] Taype-Rondan A. et al., Smoking and heavy drinking patterns in rural, urban and rural-tourban migrants: the PERU MIGRANT Study. BMC Public Health 17, 165 (2017).

[25] Carrillo-Larco R. M. et al., Cohort Profile: The PERU MIGRANT Study-A prospective cohort study of rural dwellers, urban dwellers and ruralto-urban migrants in Peru. Int J Epidemiol 46, 1752-1752f (2017).

[26] D. Matthew, Scrutinizing Global Short-Term Medical Outreach. Hastings Center Report 37, 21-23 (2012).
[27] Butler M. et al., Guidelines and checklists for short-term missions in global pediatric surgery: Recommendations from the American Academy of Pediatrics Delivery of Surgical Care Global Health Subcommittee, American Pediatric Surgical Association Global Pediatric Surgery Committee, Society for Pediatric Anesthesia Committee on International Education and Service, and American Pediatric Surgical Nurses Association, Inc. Global Health Special Interest Group. Paediatr Anaesth 28, 392-410 (2018).

[28] Roche S. D., Ketheeswaran P., and Wirtz V. J., International short-term medical missions: a systematic review of recommended practices. Int J Public Health 62, 31-42 (2017).

[29] Mach J. C., Barone H., Boni C., Jimenez H., and Tinglin M., Evaluating the impact of an international short-term medical mission through diabetic glycemic control. J Public Health (Oxf) (2018).

[30] G. L. Matlick, Short-Term Medical Missions: Toward an Ethical Approach. Am J Nurs 118, 11 (2018).

[31] J. B. Oliphant, Short-Term Medical Missions Done Well: What Every Sponsoring Institution Should Understand. J Physician Assist Educ 29, 58-61 (2018).

[32] Lasker J. N. et al., Guidelines for responsible short-term global health activities: developing common principles. Globalization and Health 14, 18 (2018).

Citation: Franklin N \& Quian A, Choi H, Valdivieso M, Lam SK, Urban Versus Rural: A Cross-Sectional Study of a Successful Peruvian Global Health Medical Mission. ARC Journal of Public Health and Community Medicine.2019; 4(3):30-36. DOI: dx.doi. org/10.20431/2456-0596.0403004.

Copyright: (c) 2019 Authors. This is an open-access article distributed under the terms of the Creative Commons Attribution License, which permits unrestricted use, distribution, and reproduction in any medium, provided the original author and source are credited. 